\title{
Aortic stenosis and anemia with an update on approaches to managing angiodysplasia in 2018
}

\author{
Kevin Mohee ${ }^{1}$, Omar Aldalati ${ }^{2}$, Rafal Dworakowski ${ }^{2,3}$, Hasan Haboubi ${ }^{4}$ \\ ${ }^{1}$ Department of Cardiology, ABMU Health Board, Morriston Hospital, Swansea, United Kingdom \\ ${ }^{2}$ Kings College London, London, United Kingdom \\ ${ }^{3}$ Department of Cardiology, Medical University of Gdansk, Poland \\ ${ }^{4}$ Swansea University, Swansea, United Kingdom
}

\begin{abstract}
Angiodyplasia and aortic stenosis are both conditions that are highly prevalent in elderly people and can often co-exist. Recent studies suggest that this association is related to subtle alterations in plasma coagulation factors. The von Willebrand factor is the strongest link between aortic stenosis and bleeding associated with gastrointestinal angiodysplasia. With an ageing population, the disease burden of aortic stenosis and its association with angiodysplasia of the bowel makes this an incredibly underdiagnosed yet important condition. Clinicians should be aware of this association when dealing with elderly patients presenting either with unexplained anemia, gastrointestinal bleeding or with aortic stenosis. A high index of suspicion and appropriate diagnostic techniques followed by appropriate and prompt treatment could be life-saving. No clear guidelines exist on management but surgical aortic valve replacement is thought to offer the best hope for long-term resolution of bleeding. With a growing number of technological armamentarium in the management of such patients, especially with the advent of transcatheter aortic valve implantation, new options can be offered even to elderly patients with comorbidities for whom conventional surgery would have been impossible. (Cardiol J 2020; 27, 1: 72-77)
\end{abstract}

Key words: aortic stenosis, Heyde's syndrome, angiodysplasia, von Willebrand factor, transcatheter aortic valve implantation

\section{Introduction}

Angiodysplasia is one of the commonest reported causes of gastrointestinal (GI) bleeding (GIB) in the elderly population [1]. It is an acquired submucosal arterio-venous malformation that arises during the ageing process due to the combination of high stress and deficiency of collagen IV that supports the connective tissue in the GI wall [2]. This condition is often associated with aortic stenosis (AS) and may be referred to by its eponymous name, Heyde's syndrome [1]. There remains an ongoing debate as to whether there is a genuine association or it is just coincidental because the mechanism connecting both pathologies remains unclear. There is however, a general consensus that aortic valve replacement (AVR) in this setting terminates GI blood loss in the majority of patients [3].

This paper aims at reviewing the evidence between aortic stenosis and anaemia with a particular focus on angiodysplasia.

\section{Subheadings}

\section{Historical perspective}

Heyde's syndrome was first described in 1958 by Edward Heyde following a series of 10 patients with AS and GI bleeding of unknown origin [1]. Subsequent reports have inferred GI angiodysplasia especially in the right colon as a probable site of the bleeding but the exact association between AS and angiodysplasia remained unclear. Later, angiodysplasia of the stomach and duodenum was

Address for correspondence: Rafal Dworakowski, MD, PhD, Department of Cardiology, King's College Hospital, Denmark Hill, London, SE5 9RS, United Kingdom, tel: ++44-20-3299-3379, e-mail: rdworakowski@nhs.net 
reported in patients with AS as being a potential cause of recurrent unexplained massive upper GI hemorrhage. With the combined use of both angiography and endoscopy to identify GI lesions, AS associated with angiodysplasia has been thoroughly documented, and can include both upper GI and lower GI lesions, particularly right sided colonic lesions.

It was not until 1992 that an etiopathogenic mechanism was postulated. According to Warkentin et al. [4], this phenomenon arises due to a deficiency of high molecular weight (HMW) multimeters of the von Willebrand factor (vWF). Type 2A von Willebrand disease appears to be the nexus between two components of this condition and is reported in as many as $67-92 \%$ of patients with severe AS [5].

\section{Epidemiology}

Anemia is a common finding among patients with severe AS. Rheude et al. [6] and Tjahjadi et al. [7] reported a $45 \%$ and a $30.1 \%$ prevalence of anaemia (World Health Organization definition) among patients undergoing surgical aortic valve replacement (SAVR) and transcatheter aortic valve implantation (TAVI), respectively. On the other hand, it has been postulated that the prevalence of calcific AS is much higher in patients with idiopathic GIB compared to non-idiopathic GIB and the general population. Several studies have reported a prevalence of $7-41 \%$ of AS with symptomatic GIB [7-10].

Godino et al. [11] elegantly showed that among 400 consecutive TAVI patients (Nov 2007 till Feb 2012), 37 patients had a history of GIB (9.2\%). Of those, 7 (1.7\%) patients had confirmed instrumental evidence of angiodysplasia and a final diagnosis of Heyde's syndrome.

\section{Pathophysiology}

Several pathological mechanisms have been hypothesized to explain this condition and these include an age-related degenerative process, genetic predisposition and hematological abnormalities.

\section{Age related degenerative process}

This condition presents in patients of advanced age, usually in the seventh decade of life (range 53-90 years old) [11]. The bleeding is normally recurrent and potentially massive. Age dependant tissue deterioration is a known risk factor of both
AS and angiodysplasia and the incidence of both increases with age. Hence, angiodysplasia was thought to be due to senescence rather than AS per se [11].

\section{Genetic predisposition}

Heyde's syndrome may be attributed to a common genetic predisposition for an underlying defect in connective tissue disease, particularly a deficiency of collagen type IV. Congenital connective tissue defects may aggravate age dependant tissue degradation essentially due to high tension and weak supporting tissue particularly in the colon [12]. Likewise, congenital abnormalities of the aortic valve, namely the bicuspid valve are other risk factors to induce calcification of the aortic valve [13].

\section{Hematological abnormalities}

Hematological disorders, especially defects of vWF can play a key role in Heyde's syndrome [14]. vWF is a multimeric glycoprotein that controls adhesion of platelets to the subendothelium of damaged blood vessels. Any cardiovascular disease that induces rapid clearance of HMW multimers (multimers of $\mathrm{vWF} \geq 10$ dimers are known as HMW multimers) could cause bleeding from angiodysplasia. It is therefore understandable that the high shear in vasculature present in patients with AS is responsible for the decrease in size of the vWF mulitimers. Conversely, substantial improvement is noted in the amount of HMW multimers after surgical valve replacement [5] and TAVI $[15,16]$.

\section{Other}

Boss and Rosenbaum [16] described distension of intestinal mucosal vessels in post-mortem subjects with AS as the cause of GI blood loss. This reflex vasodilation is believed to be caused via the sympathetic system due to low-grade hypoxia. There is relaxation of the vascular smooth muscle, which with chronicity results in distension. It has also been postulated that in AS there is a redistribution of the splanchnic blood flow with associated mesenteric ischemia along with high intestinal intramural pressure. Pulse wave arterial patterns in the final portion of the superior mesenteric conduit have shown a significant drop in arterial pressure in patients with AS compared to controls [17]. Ultimately the above pathological processes result in mucosal ischemia and bleeding due to loss of gut integrity. 


\section{Clinical presentation}

Most patients are elderly, more than 65 years old with known valvular heart disease or who have undergone heart valve replacement [11]. Patients with AS can present with unexplained chronic lower GIB which can cease and recur after several years. Angiodysplasia can be diagnosed as an incidental finding in an otherwise asymptomatic patient with valvular heart diseases or who has had an acute massive hemorrhage [11]. Another presentation is unexplained chronic iron deficiency [18].

\section{Diagnostic modalities}

Diagnosis remains a challenge because most patients do not exhibit generalized hemostatic deficit and bleeding is generally confined to GI tract but we believe that diagnosis should consist of a triad of cardiovascular, GI and hematological investigations.

\section{Cardiovascular investigation}

A transthoracic echocardiogram is the first line investigation to confirm the presence of AS and assess its severity.

\section{GI investigation}

Colonoscopy, either alone, or alongside angiography can be both diagnostic and therapeutic.

Whenever feasible, colonoscopy and gastroduodenoscopy are the initial diagnostic modalities of choice [19]. Selective mesenteric angiography may serve as a useful diagnostic technique for angiodysplasia, particularly in patients with a massive hemorrhage where a colonoscopic diagnosis is difficult. Before establishing a diagnosis of Heyde's syndrome in an elderly patient with unexplained or unrevealed bleeding, other diagnoses such as GI malignancy, celiac disease, vitamin B12 or folate deficiency must be excluded. However, presence of angiodysplasia on sigmoidoscopy or colonoscopy should raise suspicions of Heyde's syndrome particularly in patients with known echocardiographic evidence of AS or classical auscultatory findings of AS [19].

Wireless capsule endoscopy (CE) and double balloon enteroscopy (DBE) are newer techniques that allow diagnostic enteroscopy of the entire small intestine. $\mathrm{CE}$ is a painless, non-invasive procedure that facilitates visualization of the small intestine and indicates the route for enteroscope insertion [20]. However, CE is not a reliable method to detect all the lesions especially during active bleeding and does not offer a therapeutic intervention. DBE however can overcome the disadvantages of $\mathrm{CE}$ as it can visualize most of the small bowel by providing superior quality images compared to $\mathrm{CE}$, enables diagnostic manoeuvres and therapeutic intervention such as achieving hemostasis, performing polypectomies, stricture dilatation and stenting. Radionuclide methods such as labelled red cell scintigraphy can also be used to image the GI tract over a prolonged period as GIB in this condition, whilst episodic is usually recurrent [21]. Endoscopic Doppler sonography has also been used to identify intestinal angiodysplasias and has also been used to indicate efficacy of treatment [22].

Despite significant advances in endoscopic technology as well as standardization of training and lesion recognition, the lesion can potentially be missed in about 35\% of cases despite all investigations. Diagnostic laparotomy could be the only option and may be life-saving in such cases [23].

\section{Hematological investigations}

Heyde's syndrome is usually associated with one of the acquired forms of vWF deficiency and the recommended guidelines for diagnosis and management which involves measurement of vWF levels. Immunoblot analysis or gel electrophoresis can detect a low level of HMW vWF multimers. $\mathrm{vWF}$ antigen levels and ristocetin co-factor activity are abnormal when where is severe deficit of the largest vWF multimers [24]. Likewise, patients with AS have a higher incidence of prolonged bleeding time with an otherwise normal coagulation state compared to normal subjects. However, tests performed to detect von Willebrand diseases are not always confirmatory, as the abnormality may be subtle [24]. Moreover, multiple blood transfusions can also make vWF levels inconclusive [2].

\section{Management}

As of now, there are no recommended guidelines or universally accepted unified treatment or protocol for the management of Heyde's syndrome. One should consult guidelines for management of unexplained GI blood loss, AS and vWF-deficiency before treating patients with Heyde's syndrome. Therapeutic options for Heyde's syndrome include AVR, surgery, angiographic intervention, double or single balloon endoscopy and medical therapy particularly hormonal and thalidomide treatment. 


\section{Aortic valve replacement}

Aortic valve replacement is recommended as the first line treatment in this condition as it reduces or terminates GI blood loss [23]. Cessation of bleeding after AVR was found in $95 \%$ as compared to $5 \%$ managed by laparotomy with or without bowel resection [23]. Following AVR, it was also found that patients had improved levels of HMW multimers of vWF. AVR ameliorates mucosal blood supply and corrects the hematological abnormality $[23,24]$. GI angiodysplasia could cease following AVR as shown by endoscopy, hence, elective GI surgery for bleeding angiodysplasias in patients with co-existing AS needs to be rescheduled until AVR has been attempted [25]. Moreover, in the presence of chronic anemia requiring multiple blood transfusions coupled with reduced levels of vWF, AVR should be considered even in asymptomatic but hemodynamically significant AS patients [26]. Further studies are needed to clarify whether Heyde's syndrome should be considered as an indication for valve surgery in the absence of cardiac symptoms, anemia or GIB.

Valve replacement can result in almost immediate cessation of bleeding and remission of GIB, a theoretically, mechanical valve replacement warrants lifelong anticoagulation and this could promote GI bleeding from angiodysplastic lesions. Hence, biological valve replacement has been preferred over mechanical valves [27]. Some studies report no recurrence of bleeding in patients despite having had a mechanical valve with oral anticoagulant therapy. Indeed, some authors suggest that choice between a biological and a mechanical valve makes no difference so long as AS is treated promptly and effectively [28].

Of note, patient-prosthesis mismatch and structural valve deterioration usually lead to recurrence of angiodysplasia and bleeding [29].

\section{Transcatheter aortic valve implantation}

The new paradigm shift in the management of AS, after the recent update of the European and American guidelines to include TAVI as a treatment option for intermediate risk cases, means that many of these patients will undergo TAVI rather than SAVR. However, very few studies have investigated the effect of using TAVI to treat patients with coexisting HMW multimers of vWF and severe AS. Spangenberg et al. [30] enrolled 95 patients for elective TAVI of which 40 had abnormal multimers with $42 \%$ of these patients experiencing an average HMW multimers content of $16.2 \pm 3.3 \%$. TAVI led to a normalization of HMW multimers content in patients with prior abnormal multimers and also left HMW multimers in the remainder of the study population unaltered [30]. Similar findings were reported by Caspar et al. [31] and Marggraf et al. [32]. Spangenberg et al. [30] did not show any increase in bleeding of transfusion requirements in patients with severe vWF deficiency undergoing TAVI as opposed to surgical valve repair.

Based on the above studies, one can assume that TAVI is likely to be a curative option for Heyde's syndrome and its associated GIB just like SAVR. Godino et al. [11], as mentioned above, reported on 7 cases of Heyde's syndrome that underwent TAVI. Of those, one had failed TAVI due to access issues, the others had successful implants. A median follow up of $22 \pm 15$ months of this small group of patients demonstrated that no patients had recurrence of GIB and the only patient that had failed TAVI required repeated transfusions.

Para-valvular leak (PVL) post TAVI, yet again, is this treatment's Achilles' heel. PVL cases tend to have inferior HMW multimers recovery, and potentially this could abate the potential curative effect TAVI has over Heyde's syndrome [30]. Thus TAVI deserves consideration as the primary approach for patients with severe acquired von Willebrand syndrome or abnormal multimers.

\section{GI surgery/endoscopy}

Treatment of bleeding GI angiodysplasia may require surgical resection of the affected part or frequently endoscopic laser photocoagulation with good initial results but recurrence occurs in a third of patients. Previously, segmental bowel resection of angiodysplasia lesions was the recommended treatment for patients with Heyde's syndrome. In the absence of any colonic abnormalities observed intra-operatively, a blind right colectomy was recommended [33]. Ogano et al. [34] highlighted the need for elective bowel resection once a restricted bleeding site had been identified in patients at high risk of cardiac surgery. However, bleeding recurs in about a third of patients possibly due to lesions elsewhere in the GI tract which continues or starts to bleed in contrast to patients who undergo AVR who usually have long-term durable remissions [34]. GI surgery is only recommended if hemorrhage persists after a period of observation in patients who have demonstrable vascular ectasia on selective mesenteric angiography. A few case reports have described massive GI hemorrhage after SAVR, hence this strategy might not be ideal treatment in every patient with Heyde's syndrome [35]. 
In patients deemed unfit for AVR or surgical resection, endoscopic management has been the mainstay of treatment. Endoscopic destruction is usually performed using non-contact techniques with APC considered superior to Nd:YAG laser treatment due to lower risk of perforation [36].

\section{Medical management}

Replacement of clotting factors, vWF supplementations or Contact $\mathrm{F}$ (combination of vWF and factor VIII) can be effective for acute control of GIB $[17,18]$. Other alternatives include desmopressin, cryoprecipitate or the pan haemostatic agent recombinant factor VIIa transfusion that can augment circulating levels of vWF [37]. Other forms of medical therapy include use of somatostatin or octreotide that potentially reduces venous pressure in the portal venous system [38, 39]. This can aid in controlling bleeding, although the long-term benefit of Octreotide is still undergoing evaluation [40]. Hormonal replacement therapy in the form of low doses of ethinyl estradiol and norethisterone can reduce transfusion requirements by $50 \%$ [41]. However, the mechanism of action behind this remains unknown.

\section{Other}

Angiographic embolization of the source artery of the bleeding site could be an option to surgery [41]. Some studies recommend this approach only for high-risk surgical patients while others argue that this procedure can be considered in all patients presenting with massive GIB. The endoscopic treatment of angiodysplasia in the form of electrocoagulation sclerotherapy and laser photocoagulation has been shown to be effective [42], however several treatment attempts may be required due to a high bleeding recurrence rate.

Thus, angiography and embolization, whilst often used as a rescue approach for the management of GIB following failed endoscopic therapy may have a larger role to play.

\section{Future work: Biomarkers predicting risk}

The vascular growth factor, angiopoeitin-2 which plays an important role in embryonic angiogenesis may also have a role as a biomarker in predicting patients at risk of bleeding. Angiopoeitin-2 levels (both serum and mucosal levels) have been found to be elevated in patients with sporadic small bowel angiodysplasia [43]. It remains to be seen whether these levels are affected by AVR.

\section{Conclusions}

The nexus between aortic valve stenosis and GIB still remains poorly understood. Its potentially life-threatening nature and uncertainty renders it a difficult condition to treat. However, a high index of suspicion must alert any clinician especially in the emergency department while treating an elderly patient with co-existing gastrointestinal hemorrhage and AS. A multi-disciplinary approach can be comprised of a gastroenterologist, cardiologist, cardiac surgeon and a hematologist to best treat such patients. Besides, further studies must be carried out to explore the pathophysiology and management options for this condition.

\section{Conflict of interest: None declared}

\section{References}

1. Goligher J. Angiodyslasia. Surgery of the anus, rectum and colon. Bailliere Tindall, London 1984: 1058-1074.

2. Luckraz H, Hashim S, Ashraf S. Aortic stenosis and angiodysplasia in the elderly: common things occur commonly? Interact Cardiovasc Thorac Surg. 2003; 2(4): 526-528, doi: 10.1016/ S1569-9293(03)00128-2, indexed in Pubmed: 17670112.

3. Bhutani MS, Gupta SC, Markert RJ, et al. A prospective controlled evaluation of endoscopic detection of angiodysplasia and its association with aortic valve disease. Gastrointest Endosc. 1995; 42(5): 398-402, indexed in Pubmed: 8566626.

4. Warkentin TE, Moore JC, Morgan DG. Aortic stenosis and bleeding gastrointestinal angiodysplasia: is acquired von Willebrand's disease the link? Lancet. 1992; 340(8810): 35-37, indexed in Pubmed: 1351610.

5. Vincentelli A, Susen S, Le Tourneau T, et al. Acquired von Willebrand syndrome in aortic stenosis. N Engl J Med. 2003; 349(4): 343-349, doi:10.1056/NEJMoa022831, indexed in Pubmed: 12878741.

6. Rheude T, Pellegrini C, Michel J, et al. Prognostic impact of anemia and iron-deficiency anemia in a contemporary cohort of patients undergoing transcatheter aortic valve implantation. Int J Cardiol. 2017; 244: 93-99, doi: 10.1016/j.ijcard.2017.06.024, indexed in Pubmed: 28645804.

7. Tjahjadi C, Wee Y, Hay K, et al. Heyde syndrome revisited: anaemia and aortic stenosis. Intern Med J. 2017; 47(7): 814-818, doi: 10.1111/imj.13419, indexed in Pubmed: 28276127.

8. Shoenfeld Y, Eldar M, Bedazovsky B, et al. Aortic stenosis associated with gastrointestinal bleeding. A survey of 612 patients. Am Heart J. 1980; 100(2): 179-182, indexed in Pubmed: 6967691.

9. Heer M, Sulser H, Hany A. Angiodysplasia of the colon: an expression of occlusive vascular disease. Hepatogastroenterology. 1987; 34(3): 127-131, indexed in Pubmed: 2440788.

10. Rogers BH. Endoscopic diagnosis and therapy of mucosal vascular abnormalities of the gastrointestinal tract occurring in elderly patients and associated with cardiac, vascular, and pulmonary disease. Gastrointest Endosc. 1980; 26(4): 134-138, indexed in Pubmed: 6969679.

11. Godino C, Lauretta L, Pavon AG, et al. Heyde's syndrome incidence and outcome in patients undergoing transcatheter aortic valve implantation. J Am Coll Cardiol. 2013; 61(6): 687-689, doi: 10.1016/j.jacc.2012.10.041, indexed in Pubmed: 23391203. 
12. Weaver GA, Alpern HD, Davis JS, et al. Gastrointestinal angiodysplasia associated with aortic valve disease: part of a spectrum of angiodysplasia of the gut. Gastroenterology. 1979; 77(1): 1-11, indexed in Pubmed: 312746.

13. Lewin MB, Otto CM. The bicuspid aortic valve: adverse outcomes from infancy to old age. Circulation. 2005; 111(7): 832834, doi:10.1161/01.CIR.0000157137.59691.0B, indexed in Pubmed: 15723989

14. Sadler JE. Biochemistry and genetics of von Willebrand factor. Annu Rev Biochem. 1998; 67: 395-424, doi: 10.1146/annurev. biochem.67.1.395, indexed in Pubmed: 9759493.

15. Sedaghat A, Kulka H, Sinning JM, et al. Transcatheter aortic valve implantation leads to a restoration of von Willebrand factor (VWF) abnormalities in patients with severe aortic stenosis - Incidence and relevance of clinical and subclinical VWF dysfunction in patients undergoing transfemoral TAVI. Thromb Res. 2017; 151: 23-28, doi: 10.1016/j.thromres.2016.12.027, indexed in Pubmed: 28088607.

16. Boss EG, Rosenbaum JM. Bleeding from the right colon associated with aortic stenosis. Am J Dig Dis. 1971; 16(3): 269-275, indexed in Pubmed: 5313835.

17. Leimbach WN, Marsidi I, Leininger NR, et al. Aortic stenosis and intestinal angiodysplasia--a case of gastric involvement. West J Med. 1981; 135(2): 139-142, indexed in Pubmed: 6974434.

18. Singh P, Scoyni R, Pooran N, et al. Aortic valve replacement: a last resort for aortic stenosis-associated refractory GI bleeding. Gastrointest Endosc. 2002; 56(1): 139-141, indexed in Pubmed: 12085055.

19. Kameda N, Higuchi K, Shiba M, et al. A prospective, single-blind trial comparing wireless capsule endoscopy and double-balloon enteroscopy in patients with obscure gastrointestinal bleeding. J Gastroenterol. 2008; 43(6): 434-440, doi: 10.1007/s00535-0082182-9, indexed in Pubmed: 18600387.

20. Anderson RP, McGrath K, Street A. Reversal of aortic stenosis, bleeding gastrointestinal angiodysplasia, and von Willebrand syndrome by aortic valve replacement. Lancet. 1996; 347(9002): 689-690, indexed in Pubmed: 8596401.

21. Jaspersen D, Körner T, Schorr W, et al. Diagnosis and treatment control of bleeding colorectal angiodysplasias by endoscopic Doppler sonography: a preliminary study. Gastrointest Endosc. 1994; 40(1): 40-44, indexed in Pubmed: 8163133.

22. Ogano M, Iwasaki Yk, Takano H, et al. Successful colectomy for the treatment of repetitive bleeding from colonic angiodysplasia in a patient with Heyde syndrome. Intern Med. 2006; 45(6): 355-358, indexed in Pubmed: 16617184.

23. Andersen MR, Aaseby J. Somatostatin in the treatment of gastrointestinal bleeding caused by angiodysplasia. Scand J Gastroenterol. 1996; 31(10): 1037-1039, indexed in Pubmed: 8898427.

24. Soran H, Lewis M, Whorwell PJ. Bleeding angiodysplasia: should we concentrate more on the aortic valve than on the bowel? Int J Clin Pract. 2002; 56(2): 155-156, indexed in Pubmed: 11926706.

25. Scheffer SM, Leatherman LL. Resolution of Heyde's syndrome of aortic stenosis and gastrointestinal bleeding after aortic valve replacement. Ann Thorac Surg. 1986; 42(4): 477-480, indexed in Pubmed: 3490235.

26. Varma P, Misra M, Radhakrishnan VV, et al. Fatal post-operative gastro intestinal hemorrhage because of angio-dysplasia of small intestine in aortic regurgitation. Interact Cardiovasc Thorac Surg. 2004; 3(1): 118-120, doi: 10.1016/S1569-9293(03)00233-0, indexed in Pubmed: 17670193.

27. Cappell MS, Lebwohl O. Cessation of recurrent bleeding from gastrointestinal angiodysplasias after aortic valve replacement. Ann Intern Med. 1986; 105(1): 54-57, indexed in Pubmed: 3487267.

28. Roberts WC, Ko JMi, Hamilton C, et al. Frequency by decades of unicuspid, bicuspid, and tricuspid aortic valves in adults having isolated aortic valve replacement for aortic stenosis, with or without associated aortic regurgitation. Circulation. 2005; 111(7): 920-925, doi:10.1161/01.CIR.0000155623.48408.C5, indexed in Pubmed: 15710758.
29. Apostolakis E, Doering C, Kantartzis M, et al. Calcific aorticvalve stenosis and angiodysplasia of the colon: Heyde's syndrome--report of two cases. Thorac Cardiovasc Surg. 1990; 38(6): 374-376, doi: 10.1055/s-2007-1014055, indexed in Pubmed: 2291237.

30. Spangenberg T, Budde U, Schewel D, et al. Treatment of acquired von Willebrand syndrome in aortic stenosis with transcatheter aortic valve replacement. JACC Cardiovasc Interv. 2015; 8(5): 692-700, doi: 10.1016/j.jcin.2015.02.008, indexed in Pubmed: 25946442

31. Caspar T, Jesel L, Desprez D, et al. Effects of transcutaneous aortic valve implantation on aortic valve disease-related hemostatic disorders involving von Willebrand factor. Can J Cardiol. 2015; 31(6): 738-743, doi: 10.1016/j.cjca.2015.01.012, indexed in Pubmed: 25935884.

32. Marggraf O, Schneppenheim S, Daubmann A, et al. Correction of acquired von Willebrand syndrome by transcatheter aortic valve implantation. J Invasive Cardiol. 2014; 26(12): 654-658, indexed in Pubmed: 25480995.

33. Warkentin TE, Moore JC, Anand SS, et al. Gastrointestinal bleeding, angiodysplasia, cardiovascular disease, and acquired von Willebrand syndrome. Transfus Med Rev. 2003; 17(4): 272-286, indexed in Pubmed: 14571395.

34. Ogano M, Iwasaki Yk, Takano H, et al. Successful colectomy for the treatment of repetitive bleeding from colonic angiodysplasia in a patient with Heyde syndrome. Intern Med. 2006; 45(6): 355-358, indexed in Pubmed: 16617184.

35. Meyer CT, Troncale FJ, Galloway S, et al. Arteriovenous malformations of the bowel: an analysis of 22 cases and a review of the literature. Medicine (Baltimore). 1981; 60(1): 36-48, indexed in Pubmed: 6969839.

36. Dray X, Camus M, Coelho J, et al. Treatment of gastrointestinal angiodysplasia and unmet needs. Dig Liver Dis. 2011; 43(7): 515-522, doi: 10.1016/j.dld.2010.12.007, indexed in Pubmed: 21239239 .

37. Meijer K, Peters FT, van der Meer J. Recurrent severe bleeding from gastrointestinal angiodysplasia in a patient with von Willebrand's disease, controlled with recombinant factor VIIa. Blood Coagul Fibrinolysis. 2001; 12(3): 211-213, indexed in Pubmed: 11414636.

38. Andersen MR, Aaseby J. Somatostatin in the treatment of gastrointestinal bleeding caused by angiodysplasia. Scand J Gastroenterol. 1996; 31(10): 1037-1039, indexed in Pubmed: 8898427.

39. Bowers M, McNulty O, Mayne E. Octreotide in the treatment of gastrointestinal bleeding caused by angiodysplasia in two patients with von Willebrand's disease. Br J Haematol. 2000; 108(3): 524-527, indexed in Pubmed: 10759709.

40. Grooteman KV, van Geenen EJM, Drenth JPH. Multicentre, open-label, randomised, parallel-group, superiority study to compare the efficacy of octreotide therapy $40 \mathrm{mg}$ monthly versus standard of care in patients with refractory anaemia due to gastrointestinal bleeding from small bowel angiodysplasias: a protocol of the OCEAN trial. BMJ Open. 2016; 6(9): e011442, doi: 10.1136/bmjopen-2016-011442, indexed in Pubmed: 27619827.

41. Granieri R, Mazzulla JP, Yarborough GW. Estrogen-progesterone therapy for recurrent gastrointestinal bleeding secondary to gastrointestinal angiodysplasia. Am J Gastroenterol. 1988; 83(5): 556-558, indexed in Pubmed: 3259072.

42. Machicado GA, Jensen DM. Endoscopic diagnosis and treatment of severe lower gastrointestinal bleeding. Indian J Gastroenterol. 2006; 25(Suppl. 1): S43-S51.

43. Holleran G, Hall B, O'Regan M, et al. Expression of Angiogenic Factors in Patients With Sporadic Small Bowel Angiodysplasia. J Clin Gastroenterol. 2015; 49(10): 831-836, doi: 10.1097/ /MCG.0000000000000260, indexed in Pubmed: 25319741. 